\title{
RASSF5 inhibits growth and invasion and induces apoptosis in osteosarcoma cells through activation of MST1/LATS1 signaling
}

\author{
XU-HUI ZHOU ${ }^{1}$, CHAO-QUN YANG $^{1}$, CHENG-LIN ZHANG $^{1}$, YANG GAO $^{1}$, HONG-BIN YUAN $^{2}$ and CE WANG ${ }^{1}$ \\ Departments of ${ }^{1}$ Orthopedic Surgery and ${ }^{2}$ Anesthesiology, Changzheng Hospital, \\ Second Military Medical University, Shanghai 200003, P.R. China
}

Received May 13, 2014; Accepted July 9, 2014

DOI: $10.3892 /$ or.2014.3387

\begin{abstract}
Ras association (RalGDS/AF-6) domain family member RASSF5 has been implicated in a variety of key biological processes, including cell proliferation, cell cycle regulation and apoptosis. It is believed to play an important role in tumorigenesis as a tumor suppressor in a number of malignancies. Yet, little is known concerning the function and underlying mechanisms of RASSF5 in human osteosarcoma (OS). The expression of RASSF5 was examined by immunohistochemical assay using a tissue microarray in 45 cases of OS tissues. A gain-of-function approach was used to observe the effects of lentiviral vector-mediated overexpression of RASSF5 (Lv-RASSF5) on cell growth, invasion and apoptosis, respectively, as indicated by MTT, Transwell and flow cytometry assays, and the expression levels of mammalian sterile 20-like (MST1) kinase, large tumor suppressor 1 (LATS1), proliferating cell nuclear antigen (PCNA), matrix metallopeptidase-9 (MMP-9) and p53 were detected by realtime PCR and western blot assays in OS cells (MG-63 and U-2 OS). The results indicated that the expression of RASSF5 protein was significantly downregulated in OS tissues compared to that in adjacent non-cancerous tissues (ANCT) (40.0 vs. $73.3 \%, P=0.002)$, and had a negative correlation with distant metastasis of the tumor $(\mathrm{P}=0.01)$. Overexpression of RASSF5 markedly suppressed cell proliferation and invasion, and induced cell apoptosis in the OS cell lines with increased expression of MST1, LATS1 and p53 and decreased expression of PCNA and MMP-9. Taken together, our findings demonstrate that RASSF5 expression is negatively correlated with distant metastasis of OS, and RASSF5 may function as a
\end{abstract}

Correspondence to: Dr Ce Wang, Department of Orthopedic Surgery, Changzheng Hospital, Second Military Medical University, 415 Fengyang Road, Shanghai 200003, P.R. China

E-mail:wc201404@163.com

Dr Hong-Bin Yuan, Department of Anesthesiology, Changzheng Hospital, Second Military Medical University, 415 Fengyang Road, Shanghai 200003, P.R. China

E-mail: yuanhongbin20144@163.com

Key words: RASSF5, osteosarcoma, growth, invasion, apoptosis tumor suppressor in OS cells through activation of the MST1/ LATS1 pathway.

\section{Introduction}

Osteosarcoma (OS), originating from bone as an aggressive bone tumor, is an infrequent but the most common and destructive primary bone tumor in children and adolescents. It is the second highest cause of cancer-related mortality, mainly due to the development of often fatal metastasis (1). In the past few decades, surgical resection therapy has resulted in the poor prognosis of OS patients. With the application of neoadjuvant chemotherapy in OS, the five-year survival rate has significantly increased $(2,3)$. To date, however, the molecular pathogenesis and etiology of OS are still not clearly elucidated.

Members of the RASSF family (RASSF1-10) have been reported to participate in a variety of important biological processes, and in particular have been identified as candidate tumor suppressors in cancers (4), of which RASSF5 (also called NORE1) is expressed in most normal tissues but is downregulated in several cancer cell lines (5). The protein expression of RASSF5 is frequently downregulated in lung tumor cell lines and primary lung tumors, impairs cell growth and mediates Ras-dependent apoptosis (6). RASSF5 has also been identified as a breakpoint-spanning gene downregulated in clear cell renal cell carcinoma (CCRCC), and inhibits cell proliferation, representing a new candidate tumor suppressor for CCRCC (7). Some studies show that no inactivating somatic mutation for RASSF5 is found in lung tumor lines, but the RASSF5 promotor region is hypermethylated in primary tumors and tumor cell lines (8). It is known that methylation of promotor $\mathrm{CpG}$ islands is a common mechanism inactivating tumor-suppressor genes in cancer. RASSF family genes including RASSF5 to various degrees are methylated in neuroblastoma cell lines and primary tumors (9). Methylation of RASSF5 frequently occurs in squamous cell carcinomas of the head and neck (10), and even has the potential for serving as a recurrence biomarker for bladder cancer (11).

However, few studies indicate that no promotor methylation of RASSF5 is detected in thyroid tumor (12) and methylation is an uncommon event in primary thyroid tumors (13). Moreover, promotor methylation is not the molecular mechanism responsible for RASSF5 suppression in neuroblastic tumors (14). 
Thus, to further clarify the function and molecular mechanism of RASSF5 in cancer, we examined the expression of RASSF5 in OS tissues by immunohistochemical (IHC) assay, and investigated the effects of RASSF5 overexpression on cell growth and invasion in vitro. We hypothesized that RASSF5 may function as a tumor suppressor implicated in the development of OS.

\section{Materials and methods}

Materials. The OS cell lines (MG-63 and U-2 OS) used for the experiments were obtained from the Institute of Biochemistry and Cell Biology (Shanghai, China). Lentiviral-mediated RASSF5 vector (Lv-RASSF5), negative control vector, and virion-packaging elements were purchased from GeneChem (Shanghai, China). Human OS tissues and the corresponding ANCT were collected from the Department of Orthopedic Surgery, Changzheng Hospital. OS tissue microarray was constructed by Shanghai Outdo Biotech Co., Ltd. (Shanghai, China). All the antibodies were purchased from Cell Signaling Technology (Boston, MA, USA). RASSF5 primer was synthesized by ABI (Framingham, MA, USA).

Drugs and reagents. Dulbecco's modified Eagle's medium (DMEM) and fetal bovine serum (FBS) were purchased from Thermo Fisher Scientific Inc. (Waltham, MA, USA); TRIzol reagent and Lipofectamine 2000 were obtained from Invitrogen (Carlsbad, CA, USA); M-MLV reverse transcriptase was purchased from Promega (Madison, WI, USA); SYBR-Green Master Mix was obtained from Takara (Otsu, Japan); and the ECL Plus kit was obtained from GE Healthcare (Piscataway, NJ, USA). Cell apoptosis kit [propidium iodide (PI), RNase A, Annexin V-FITC] was from KeyGen Biology (Nanjing, China).

Clinical samples and data. The tissue microarray was prepared for IHC test. Human OS tissues and the corresponding ANCT were obtained from biopsies in a total of 45 consecutive OS cases admitted to our hospital from January 2005 to December 2011. The baseline characteristics of the patients before neo-adjuvant chemotherapy are summarized (Table II). The study was approved by the Medical Ethics Committee of the Second Military Medical University, and written informed consent was obtained from the patients or their parents before sample collection. Two pathologists respectively reviewed all of the cases.

Tissue microarray. The Advanced Tissue Arrayer (ATA-100; Chemicon International, Temecula, CA, USA) was used to create holes in a recipient paraffin block and to acquire cylindrical core tissue biopsies with a diameter of $1 \mathrm{~mm}$ from the specific areas of the 'donor' block. The tissue core biopsies were transferred to the recipient paraffin block at defined array positions. The tissue microarrays contained tissue samples from 45 formalin-fixed paraffin-embedded cancer specimens with known diagnosis, and corresponding ANCT from these patients. The block was incubated in an oven at $45^{\circ} \mathrm{C}$ for $20 \mathrm{~min}$ to allow complete embedding of the grafted tissue cylinders in the paraffin of the recipient block, and then stored at $4^{\circ} \mathrm{C}$ until microtome sectioning.
IHC staining. Tissue microarray sections were processed for IHC analysis of RASSF5 protein as follows. Immunohistochemical examinations were carried out on 3-mm sections. For anti-RASSF5 IHC, unmasking was performed with $10 \mathrm{mM}$ sodium citrate buffer, $\mathrm{pH} 6.0$, at $90^{\circ} \mathrm{C}$ for $30 \mathrm{~min}$. For anti-RASSF5 IHC, antigen unmasking was not necessary. Sections were incubated in $0.03 \%$ hydrogen peroxide for $10 \mathrm{~min}$ at room temperature, to remove endogenous peroxidase activity, and then in blocking serum [0.04\% bovine serum albumin, A2153; Sigma-Aldrich, Shanghai, China and 0.5\% normal goat serum X0907; Dako Corporation, Carpinteria, CA, USA, in phosphate-buffered saline (PBS)] for $30 \mathrm{~min}$ at room temperature. Anti-RASSF5 antibody was used at a dilution of 1:200. The antibody was incubated overnight at $4^{\circ} \mathrm{C}$. Sections were then washed three times for $5 \mathrm{~min}$ in PBS. Non-specific staining was blocked with $0.5 \%$ casein and $5 \%$ normal serum for $30 \mathrm{~min}$ at room temperature. Finally, staining was developed using diaminobenzidine substrate, and sections were counterstained with hematoxylin. Normal serum or PBS was used to replace the anti-RASSF5 antibody in negative controls.

Quantification of protein expression. The expression of RASSF5 was semi-quantitatively estimated as total immunostaining scores, which were calculated as the product of a proportion score and an intensity score. The proportion and intensity of the staining were evaluated independently by two observers. The proportion score reflected the fraction of positive staining cells ( 0 , none; $1,<10 \% ; 2,10$ to $\geq 25 \% ; 3,>25$ to $50 \%$; and $4,>50 \%$ ), and the intensity score represented the staining intensity $(0$, no staining; 1 , weak; 2 , intermediate; and 3, strong). Finally, a total expression score was obtained ranging from 0 to 12 . Based on the analysis in advance, RASSF5 expression was categorized into two groups: lowlevel RASSF5 expression (score 0-3) and high-level RASSF5 expression (score 4-12). The scoring was independently assessed by two pathologists.

Cell culture and transfection. OS cells were cultured in DMEM supplemented with 10\% heat-inactivated FBS, $100 \mathrm{U} /$ $\mathrm{ml}$ of penicillin, and $100 \mu \mathrm{g} / \mathrm{ml}$ of streptomycin. Cells in this medium were placed in a humidified atmosphere containing $5 \% \mathrm{CO}_{2}$ at $37^{\circ} \mathrm{C}$. Cells were subcultured at a 1:5 dilution in medium containing $300 \mu \mathrm{g} / \mathrm{ml} \mathrm{G} 418$ (an aminoglycoside antibody; a commonly used stable transfection reagent in molecular genetic testing). On the day of transduction, OS cells were replated at $5 \times 10^{4}$ cells/well in 24-well plates containing serum-free growth medium with Polybrene $(5 \mathrm{mg} / \mathrm{ml})$. When reaching $50 \%$ confluency, the cells were transfected with recombinant experimental virus or control virus at the optimal multiplicity of infection (MOI) of 50 , and cultured at $37^{\circ} \mathrm{C}$ and $5 \% \mathrm{CO}_{2}$ for $4 \mathrm{~h}$. Then the supernatant was discarded and serum containing growth medium was added. At 4 days of post-transduction, transfection efficiency was measured by the frequency of green fluorescent protein (GFP)-positive cells. Positive and stable transfectants were selected and expanded for further study. The Lv-RASSF5 vector-infected clone, the negative control vector-infected and untreated OS cells were respectively named as the Lv-RASSF5, NC and control (CON) group. 
Quantitative real-time PCR. To quantitatively determine the mRNA expression level of RASSF5 in OS cells, real-time PCR was performed. Total RNA was extracted from each clone using TRIzol according to the manufacturer's protocol. Reverse transcription was carried out using M-MLV and cDNA amplification was performed using the SYBR-Green Master Mix kit according to the manufacturer's guidelines. The RASSF5 gene was amplified using a specific oligonucleotide primer, and the $\beta$-actin gene was used as an endogenous control. The PCR primer sequences were as follows: RASSF5, 5'-TTAGGAAAGAGGAATATTTTAT-3' and 5'-TAAACCTT CAACCCTACCTCTTTC-3'; $\beta$-actin, 5'-CAACGAATTTGG CTACAGCA-3' and 5'-AGGGGTCTACATGGCAACTG-3'. Data were analyzed using the comparative $\mathrm{Ct}$ method $\left(2^{-\Delta \Delta \mathrm{Ct}}\right)$. Three separate experiments were performed for each clone.

Western blot assay. OS cells were harvested and extracted using lysis buffer (Tris-HCl, SDS, mercaptoethanol and glycerol). Cell extracts were boiled for $5 \mathrm{~min}$ in loading buffer, and then an equal amount of cell extracts was separated on $15 \%$ SDS-PAGE gels. Separated protein bands were transferred onto polyvinylidene fluoride (PVDF) membranes, which were subsequently blocked in 5\% skim milk powder. Primary antibodies against RASSF5, p-MST1, p-LATS1, PCNA, MMP-2 and p53 were diluted according to the manufacturer's instructions and incubated overnight at $4^{\circ} \mathrm{C}$. Subsequently, horseradish peroxidase-linked secondary antibodies were added at a dilution of 1:1,000 and incubated at room temperature for $2 \mathrm{~h}$. The membranes were washed 3 times with PBS, and the immunoreactive bands were visualized using the ECL Plus kit according to the manufacturer's instructions. The relative protein levels in the different cell lines were normalized to the concentration of $\beta$-actin. Three separate experiments were performed for each clone.

Cell proliferation assay. Cell proliferation was analyzed using the MTT assay. Briefly, cells infected with Lv-RASSF5 viruses were incubated in 96-well plates at a density of $1 \times 10^{5}$ cells/well with DMEM supplemented with $10 \%$ FBS. Cells were treated with $20 \mu \mathrm{l}$ of MTT dye at $0,24,48$ and $72 \mathrm{~h}$, and subsequently incubated with $150 \mu \mathrm{l}$ of DMSO for $5 \mathrm{~min}$. The color reaction was measured at $570 \mathrm{~nm}$ using an enzyme immunoassay analyzer (Bio-Rad, Hercules, CA, USA). The proliferation activity was calculated for each clone.

Transwell invasion assay. Transwell filters were coated with Matrigel $(3.9 \mu \mathrm{g} / \mu \mathrm{l} ; 60-80 \mu \mathrm{l})$ on the upper surface of a polycarbonate membrane (diameter, $6.5 \mathrm{~mm}$; pore size, $8 \mu \mathrm{m}$ ). After incubating at $37^{\circ} \mathrm{C}$ for $30 \mathrm{~min}$, the Matrigel solidified and served as the extracellular matrix for analysis of tumor cell invasion. Harvested cells $\left(1 \times 10^{5}\right)$ in $100 \mu$ l of serum-free DMEM were added into the upper compartment of the chamber. A total of $200 \mu \mathrm{l}$ of conditioned medium derived from NIH3T3 cells was used as a source of chemoattractant, which was placed in the bottom compartment of the chamber. After $24 \mathrm{~h}$ of incubation at $37^{\circ} \mathrm{C}$ with $5 \% \mathrm{CO}_{2}$, the medium was removed from the upper chamber. The non-invaded cells on the upper side of the chamber were scraped off with a cotton swab. Cells that had migrated from the Matrigel into the pores of the inserted filter were fixed with $100 \%$ methanol, stained with hematoxylin,

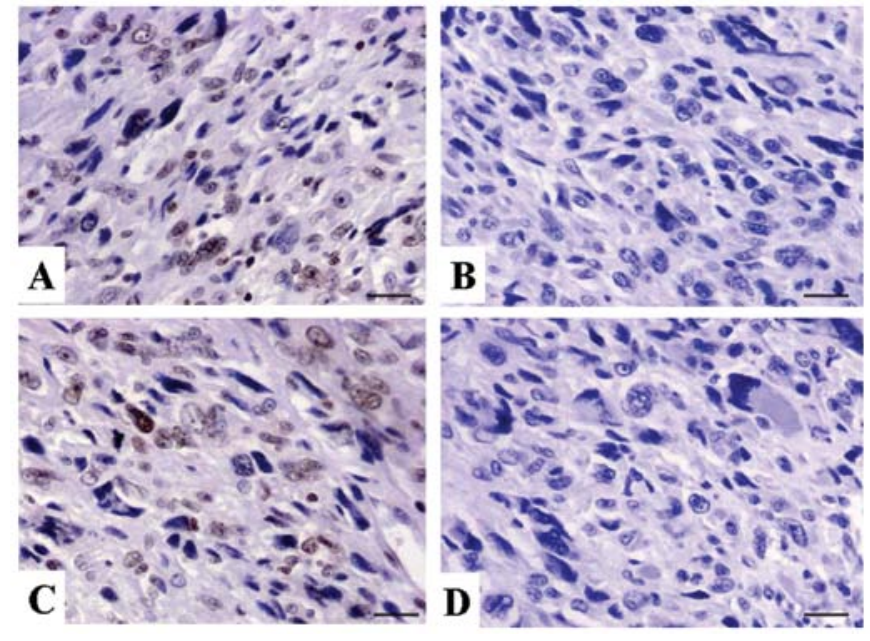

Figure 1. Expression of RASSF5 protein in osteosarcoma tissues (magnification $\mathrm{x} 400$ ). Adjacent non-cancer tissues were immunohistochemically stained with an anti-RASSF5 antibody and classified as positive expression (A) and negative expression (B). Osteosarcoma tissues were immunohistochemically stained with an anti-RASSF5 antibody and classified as positive expression (C) and negative expression (D). Positive immunostaining of RASSF5 was mainly localized in the nucleus of tumor tissue cells.

then mounted and dried at $80^{\circ} \mathrm{C}$ for $30 \mathrm{~min}$. The number of cells invading through the Matrigel was counted in 3 randomly selected visual fields from the central and peripheral portion of the filter by using an inverted microscope (x200 magnification). Each assay was repeated 3 times.

Cell apoptosis analysis. To detect cell apoptosis, OS cells treated with Lv-RASSF5 were trypsinized, washed with cold PBS and resuspended in binding buffer according to the instructions of the apoptosis kit. FITC-Annexin V and PI were added to the fixed cells for $20 \mathrm{~min}$ in darkness at room temperature. Then, Annexin V binding buffer was added to the mixture before the fluorescence was measured on a FACsort flow cytometer. The cell apoptosis was analyzed using CellQuest software (Becton-Dickinson, USA). Three separate experiments were performed for each clone.

Statistical analysis. SPSS 20.0 was used for the statistical analysis. Kruskal-Wallis $\mathrm{H}$ and Chi-square tests were used to analyze the expression rate in all groups. One-way analysis of variance (ANOVA) was used to analyze the differences between groups. The LSD method of multiple comparisons was used when the probability for ANOVA was statistically significant. Statistical significance was set at $\mathrm{P}<0.05$.

\section{Results}

Expression of RASSF5 in OS tissues. The expression of RASSF5 protein was examined using IHC staining in OS tissues. As shown in Fig. 1, the level of positive expression of RASSF5 protein was detected in OS and ANCT tissues. Positive-RASSF5 immunostaining was mainly localized in the nucleus of OS tissue cells. According to the RASSF5 immunoreactive intensity, the positive expression of RASSF5 in OS tissues was significantly downregulated compared with that in $\operatorname{ANCT}(\mathrm{P}=0.002)$ (Table I). 
Table I. Expression of RASSF5 protein in the OS tissues.

\begin{tabular}{lccccccccr}
\hline & & \multicolumn{9}{c}{ RASSF5 protein (n) } & & & & \\
\cline { 3 - 6 } Target & Sample & - & + & ++ & +++ & Total & Positive rate (\%) & $\chi^{2}$ & P-value \\
\hline \multirow{2}{*}{ RASSF5 } & OS & 27 & 11 & 5 & 2 & 45 & 40.0 & & \\
& ANCT & 12 & 17 & 11 & 5 & 45 & 73.3 & 9.965 & 0.002 \\
\hline
\end{tabular}

OS, osteosarcoma; ANCT, adjacent non-cancer tissue.

Table II. Association of RASSF5 expression with clinicopathological factors of the OS patients.

\begin{tabular}{|c|c|c|c|c|}
\hline \multirow[b]{2}{*}{ Variables } & \multirow[b]{2}{*}{$\begin{array}{c}\text { Cases (n) } \\
45\end{array}$} & \multicolumn{2}{|c|}{$\begin{array}{l}\text { RASSF5 } \\
\text { expression }\end{array}$} & \multirow[b]{2}{*}{ P-value } \\
\hline & & $\begin{array}{c}- \\
27\end{array}$ & $\begin{array}{c}+ \\
18\end{array}$ & \\
\hline Total & 45 & 27 & 18 & \\
\hline Age (years) & & & & 0.901 \\
\hline$<20$ & 28 & 17 & 11 & \\
\hline$\geq 20$ & 17 & 10 & 7 & \\
\hline Gender & & & & 0.330 \\
\hline Male & 26 & 14 & 12 & \\
\hline Female & 19 & 13 & 6 & \\
\hline Histology & & & & 0.805 \\
\hline Osteoblastic & 18 & 10 & 8 & \\
\hline Chondroblastic & 15 & 9 & 6 & \\
\hline Fibroblastic & 7 & 4 & 3 & \\
\hline Others & 5 & 4 & 1 & \\
\hline Ennecking staging & & & & 0.868 \\
\hline I & 13 & 7 & 6 & \\
\hline II & 24 & 15 & 9 & \\
\hline III & 8 & 5 & 3 & \\
\hline Distant metastases & & & & 0.010 \\
\hline No & 27 & 12 & 15 & \\
\hline Yes & 18 & 15 & 3 & \\
\hline
\end{tabular}

OS, osteosarcoma.

Association between RASSF5 expression and clinicopathological characteristics. The association of RASSF5 expression with various clinicopathological factors was analyzed. As shown in Table II, decreased expression of RASSF5 was closely correlated with distant metastasis of OS patients $(\mathrm{P}=0.01)$. However, no significant correlation was found between RASSF5 expression and other factors including age, gender of the patients, and histology and Ennecking staging of the tumor $(\mathrm{P}>0.05$, respectively).

Effect of RASSF5 overexpression on the expression of MST1 and LATS1. After Lv-RASSF5 (MOI=50) was transfected into OS cells for $24 \mathrm{~h}$, the expression levels of RASSF5 mRNA
(Fig. 2A and B) and protein (Fig. 2C and D), and p-MST1 and p-LATS1 proteins (Fig. 2C and D) were detected by real-time PCR and western blot assays, indicating that, when RASSF5 expression was significantly upregulated, p-MST1 and p-LATS1 expression was also increased in the Lv-RASSF5 group when compared with the $\mathrm{NC}$ and $\mathrm{CON}$ groups in both cell lines.

Effect of RASSF5 overexpression on cell proliferation. Deregulated cell proliferation is a hallmark of cancer. To verify the effect of RASSF5 overexpression on tumor growth in OS cells, we examined cell proliferative activities by MTT assay. The results showed that RASSF5 overexpression markedly diminished the proliferative activities of $\mathrm{OS}$ cells in a time-dependent manner compared to the $\mathrm{NC}$ and $\mathrm{CON}$ groups (Fig. 3A and $\mathrm{B}, \mathrm{P}<0.01$ ). In addition, the expression level of PCNA protein, examined by western blot assay (Fig. 3C and D), was found to be significantly downregulated in the Lv-RASSF5 group when compared with the NC and $\mathrm{CON}$ groups in both cell lines.

Effect of RASSF 5 overexpression on cell invasion. To determine the effect of RASSF5 overexpression on cell invasion, a Transwell assay was performed. The invasive potential of OS cells in the Transwell assay was determined by the ability of cells to invade a matrix barrier containing laminin and type IV collagen, major components of the basement membrane. Representative micrographs of Transwell filters are shown in Fig. 4A and B. It was found that the invasive potential of OS cells was apparently weakened in the Lv-RASSF5 groups compared to that in the $\mathrm{NC}$ and $\mathrm{CON}$ groups in both cell lines $(\mathrm{P}<0.01)$ (Fig. 4C and D). In addition, the expression level of MMP-9 protein, examined by western blot assay (Fig. 4E and F), was found significantly downregulated in Lv-RASSF5 compared with the NC group.

Effect of RASSF5 overexpression on cell apoptosis. To determine whether RASSF5 overexpression influences OS cell apoptosis, flow cytometric analysis with PI/FITC-Annexin V staining was performed. It was shown that the apoptosis indices of the OS cells in the Lv-RASSF5 group were markedly higher than indices in the NC group in both cell lines (Fig. 5A-D). Additionally, p53 pathway initiates DNA repair, cell-cycle arrest, senescence and importantly, apoptosis (16). To determine whether RASSF5 overexpression regulates the expression of p53 protein, western blotting was carried out. It was found that the protein level of p53 was increased in the 


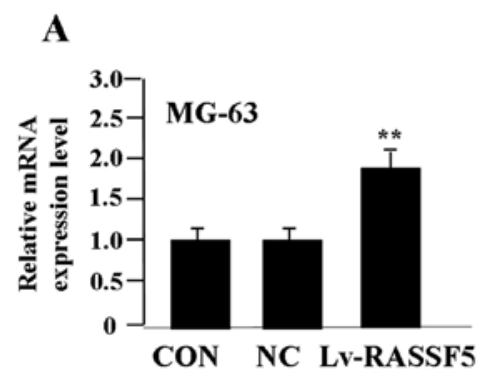

C
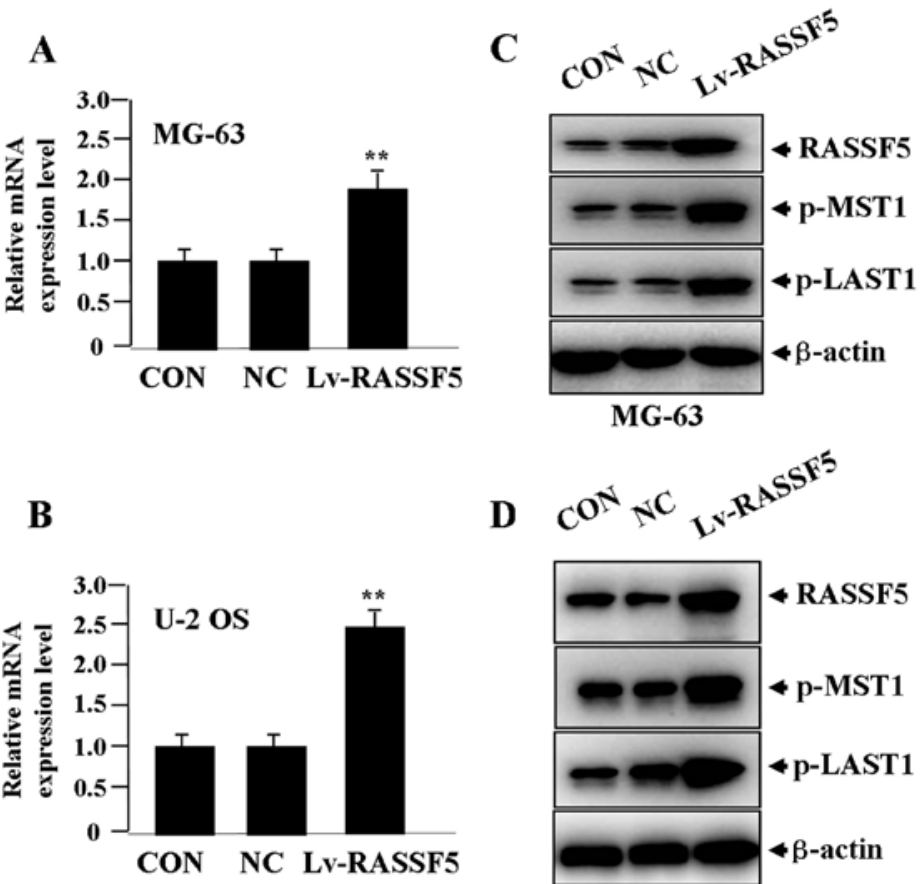

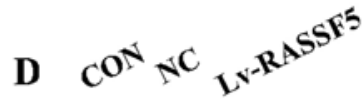

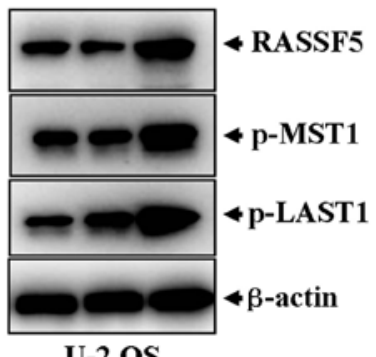

Figure 2. Effect of RASSF5 overexpression on the expression of MST1 and LATS1 in OS cells. (A and B) The mRNA expression level of RASSF5, detected by real-time PCR, was significantly increased in the Lv-RASSF5 group compared with that in the CON and NC groups in both cell lines (each ${ }^{* *} \mathrm{P}<0.01$ ). (C and D) The expression levels of p-MST1 and p-LATS1 proteins indicated by western blotting were significantly upregulated in the Lv-RASSF5 group compared with levels in the CON and NC groups in both cell lines. MST1, mammalian sterile 20-like; LATS1, large tumor suppressor 1; OS, osteosarcoma; CON, control; NC, negative control.
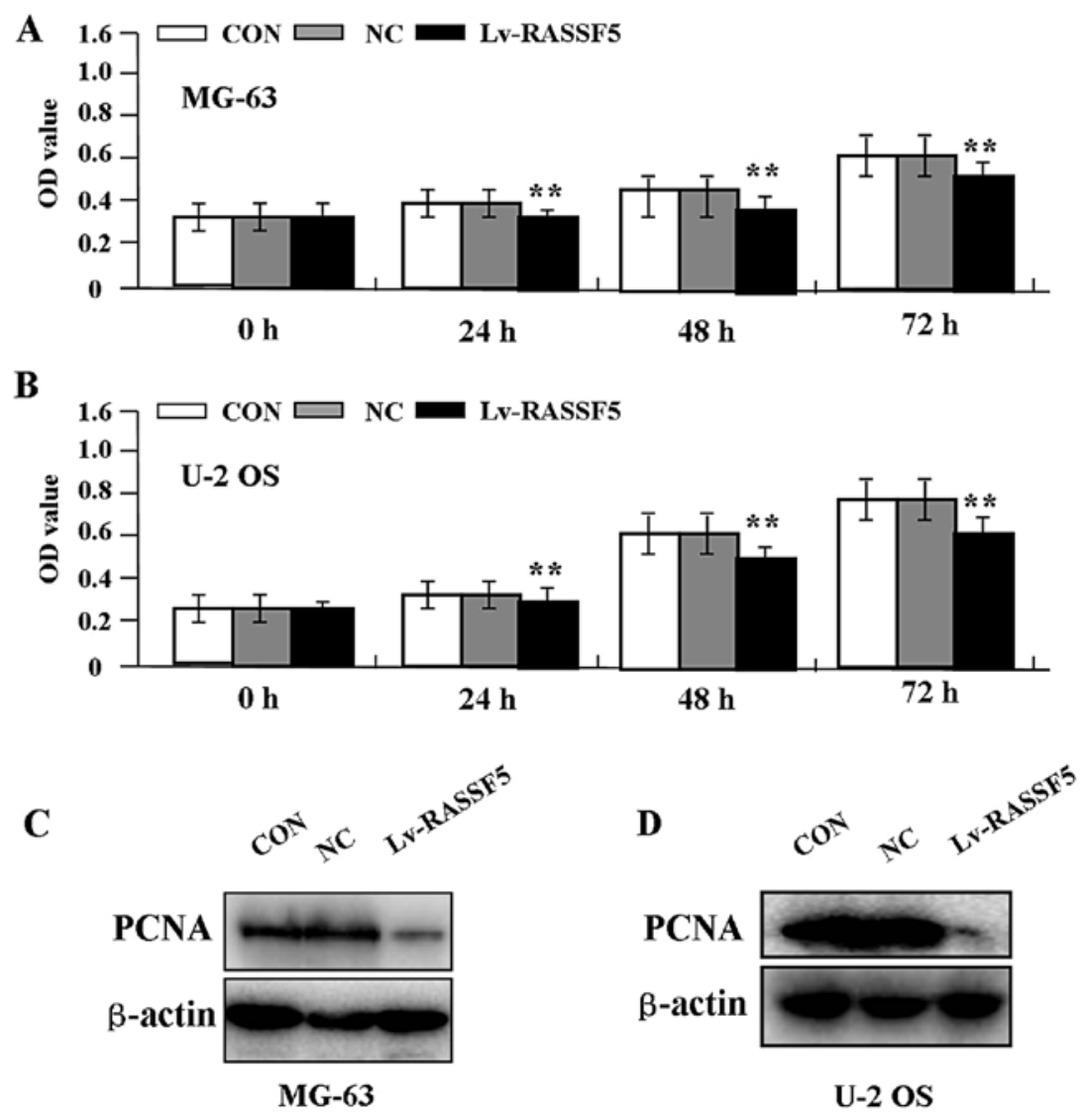

Figure 3. Effect of RASSF5 overexpression on cell proliferation of OS cells. (A and B) MTT assay was used to evaluate cell proliferative activity. Cell proliferative activity was significantly repressed in a time-dependent manner in the Lv-RASSF5 group compared with the CON and NC groups in both cell lines (each $\left.{ }^{* *} \mathrm{P}<0.01\right)$. (C and D) The expression level of PCNA protein, examined by western blot assay, was downregulated in the Lv-RASSF5 group compared with that in the $\mathrm{CON}$ and $\mathrm{NC}$ groups in both cell lines $\left({ }^{* *} \mathrm{P}<0.01\right)$. CON, control; NC, negative control; PCNA, proliferating cell nuclear antigen. 




C

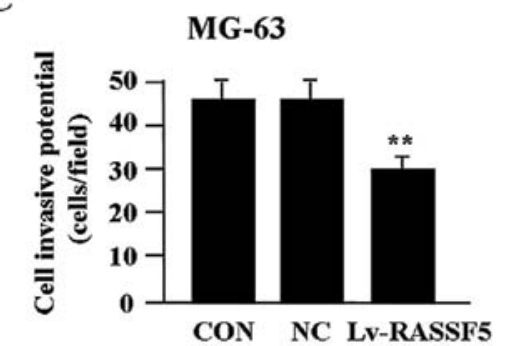

$\mathbf{E}$

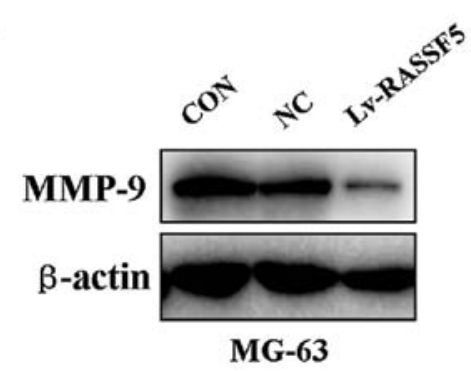

NC
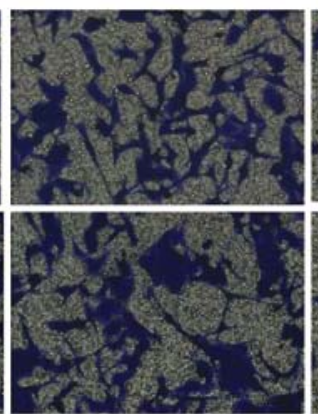

D

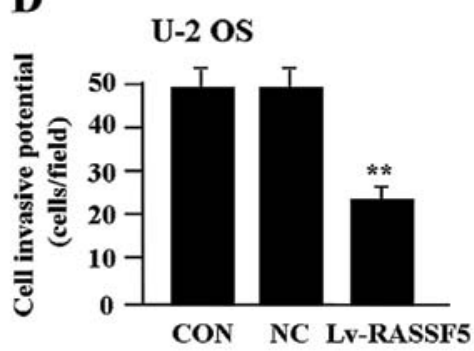

$\mathbf{F}$
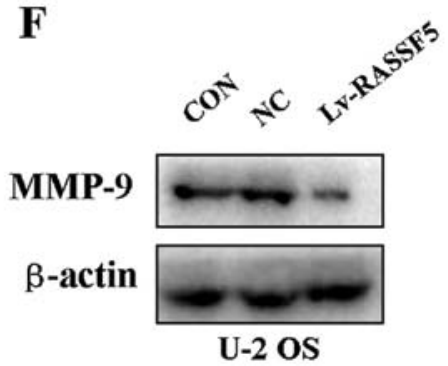

Figure 4. Effect of RASSF5 overexpression on cell invasion in OS cells. (A-D) Transwell assay was performed to determine cell invasion. Cell invasive potential was markedly weakened in the Lv-RASSF5 group compared with that in the CON and NC groups in both cell lines (** $\mathrm{P}<0.01)$. (E and F) The expression level of MMP-9 protein, examined by western blot assay, was downregulated in the Lv-RASSF5 group compared with that in the CON and NC groups in both cell lines. CON, control; NC, negative control; MMP-9, matrix metallopeptidase-9.

Lv-RASSF5 group in comparison with the NC group in both cell lines (Fig. 5E and F).

\section{Discussion}

The RASSFs comprise 10 members, RASSF1 to RASSF10, and are implicated in various cellular mechanisms including cell apoptosis, cell cycle distribution and metastases, of which RASSF5 has been reported to function as a tumor suppressor (17). Studies have shown that the candidate tumorsuppressor gene RASSF5 is epigenetically downregulated and plays a role in pathogenetic and prognostic significance in hepatocellular carcinoma (HCC) $(18,19)$. It is lowly expressed in non-small cell lung carcinoma (NSCLC) which implies a key preventive role of RASSF5 against the carcinogenesis of NSCLC (20). RASSF5 is frequently silenced mainly by aberrant promoter methylation, and is associated with tumor metastasis in CRC, implicating RASSF5 as a potential biomarker for the development of CRC (21). Yet, a limited amount of data have been reported concerning the expression of RASSF5 in OS tissues. Our present study demonstrated that RASSF5, mainly localized in the nucleus, was markedly downregulated in OS tissues when compared to the adjacent non-tumor tissues, and is negatively associated with distant metastases of the tumor, suggesting that loss of RASSF5 may represent a new biomarker involved in the development of OS.

Although RASSF5 may serve as a tumor-suppressor gene, the functions of RASSF5 are largely unknown. A loss-of-function experiment revealed that loss of RASSF5 leads to uncontrolled growth and transformation of HCC, but overexpression of RASSF5 suppresses cell replication and transformation (22). RASSF5 is suppressed in pheochromocytoma and abdominal paraganglioma, resulting in enhanced apoptosis and impaired colony formation (23). It also decreased cellular growth and induced cell apoptosis, implicated in the blockade of malignant progression of colorectal tumors (24). Thus, to further clarify the role of RASSF5 in cancer, we assessed the function of RASSF5 in the biological behaviors of OS cells, and found that overexpression of RASSF5 suppressed growth and invasion, and induced cell apoptosis in OS cells, indicating that RASSF5 may serve as a promising therapeutic target for the treatment of OS.

Furthermore, RASSF1A interacts with the pro-apoptotic kinase MST1, through activation of MST1 by promoting MST1 autophosphorylation and LATS2 phosphorylation (25), suggesting RASSF1A as part of the MST1/LATS1 signaling 
$\mathbf{A}$

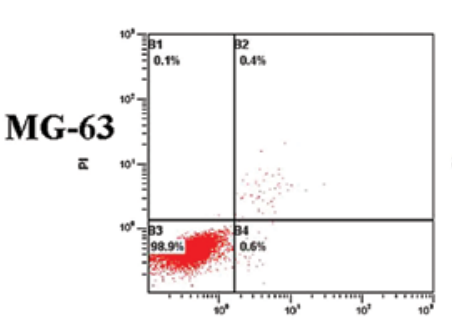

B



C

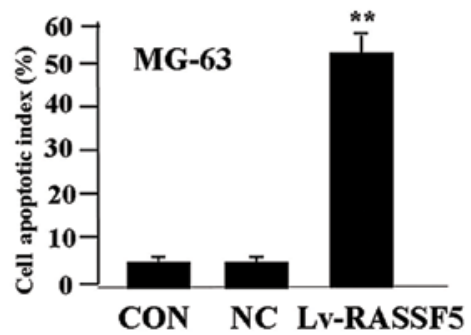

$\mathbf{E}$

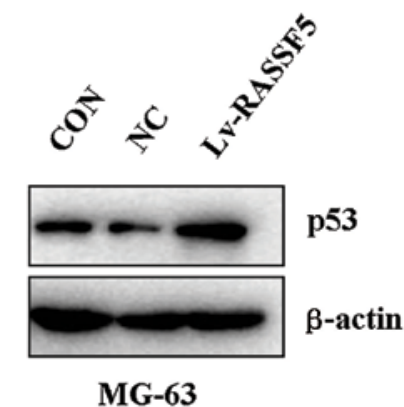

NC
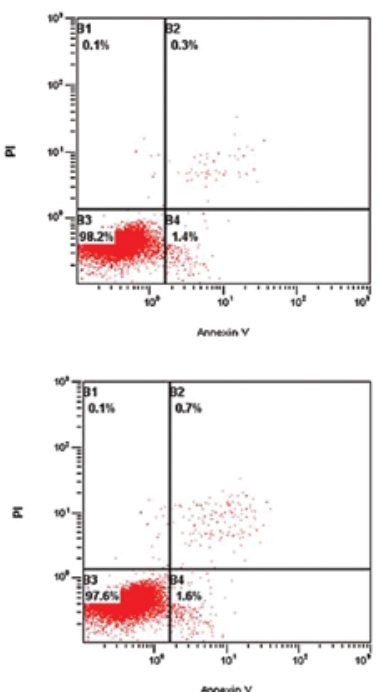

Lv-RASSF5
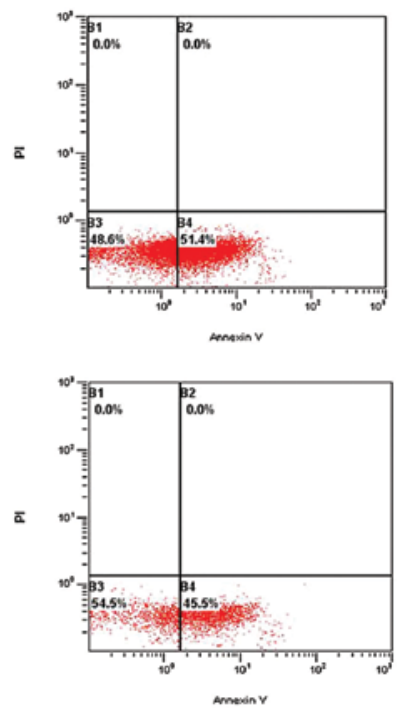

D

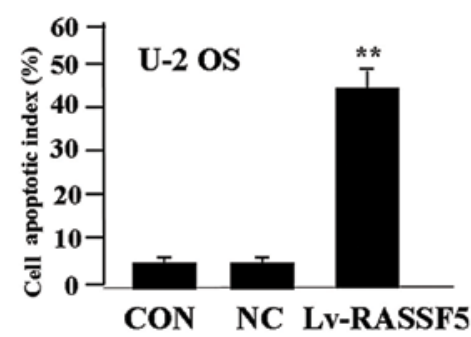

F

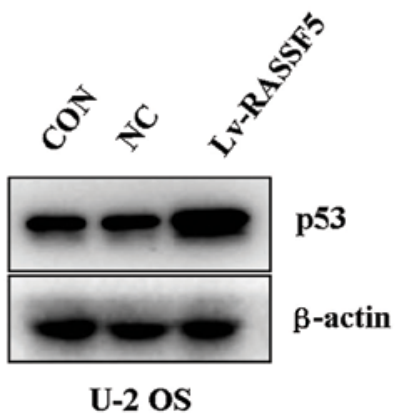

Figure 5. Effect of RASSF5 overexpression on cell apoptosis in OS cells. (A-D) Apoptotic indexes of OS cells were much higher in the Lv-RASSF5 group than those in CON and NC groups in both cell lines (** $\mathrm{P}<0.01)$. ( $\mathrm{E}$ and $\mathrm{F}$ ) The amount of p53, indicated by western blot assays, was increased in the Lv-RASSF5 group compared with that in the CON and NC groups in both cell lines. CON, control; NC, negative control. OS, osteosarcoma.

pathway, which is a major conserved mechanism governing cell contact inhibition, organ size control, and cancer development (26,27). RASSF binds to MST1, regulates mammalian cell proliferation (28), and triggers cell apoptosis via activation of mammalian MST1 (29). However, a few studies have demonstrated that MST1 exhibits a growth promoting activity in HCC cells (30), and LASSF5 suppresses the growth of lung cancer cells independent of MST1/2 kinase (31). To demonstrate the regulation of RASSF5 on MST1/LATS1 signaling in cancer, we found that RASSF5 inhibited the growth and invasion of OS cells with increased expression of p-MST1 and p-LATS1, suggesting that RASSF5 may function as a tumor suppressor in OS through activation of MST1/LATS1 signaling.

Evidence indicates that a number of biomarkers including PCNA (32), MMP-9 (33) and p53 (34) are responsible for tumor development and prognosis in a variety of cancers. MTS1 can enhance p53-dependent apoptosis of tumor cells, leading to the decreased growth of tumor cells (35). The present study revealed that overexpression of RASSF5 downregulated the expression of PCNA and MMP-9 and upregulated the expression of p-MST1, p-LATS1 and p53 in OS cells, suggesting that RASSF5 may inhibit growth and invasion via downregulation of PCNA and MMP-9 expression, and enhance pro-apoptotic effects through MST1-mediated p53 expression in OS cells.

In conclusion, our findings demonstrate that downregulation of RASSF5 expression is correlated with distant metastasis of OS tissues, and overexpression of RASSF5 may function as a tumor suppressor in OS cells through activation of the MST1/LATS1 pathway, suggesting that RASSF5 may serve as a potential therapeutic target for the treatment of OS. 


\section{References}

1. Ta HT, Dass CR, Choong PF and Dunstan DE: Osteosarcoma treatment: state of the art. Cancer Metastasis Rev 28: 247-263, 2009.

2. Tan ML, Choong PF and Dass CR: Osteosarcoma: conventional treatment vs. gene therapy. Cancer Biol Ther 8: 106-117, 2009.

3. Ottaviani $G$ and Jaffe N: The epidemiology of osteosarcoma Cancer Treat Res 152: 3-13, 2009.

4. Chan JJ, Flatters D, Rodrigues-Lima F, et al: Comparative analysis of interactions of RASSF1-10. Adv Biol Regul 53: 190-201, 2013.

5. Tommasi S, Dammann R, Jin SG, et al: RASSF3 and NORE1: identification and cloning of two human homologues of the putative tumor suppressor gene RASSF1. Oncogene 21: 2713-2720, 2002.

6. Vos MD, Martinez A, Ellis CA, et al: The pro-apoptotic Ras effector Norel may serve as a Ras-regulated tumor suppressor in the lung. J Biol Chem 278: 21938-21943, 2003.

7. Chen J, Lui WO, Vos MD, et al: The t(1;3) breakpoint-spanning genes LSAMP and NORE1 are involved in clear cell renal cell carcinomas. Cancer Cell 4: 405-413, 2003.

8. Hesson L, Dallol A, Minna JD, et al: NORE1A, a homologue of RASSF1A tumour suppressor gene is inactivated in human cancers. Oncogene 22: 947-954, 2003.

9. Djos A, Martinsson T, Kogner P and Carén H: The RASSF gene family members RASSF5, RASSF6 and RASSF7 show frequent DNA methylation in neuroblastoma. Mol Cancer 11: 40, 2012.

10. Steinmann K, Sandner A, Schagdarsurengin U and Dammann RH Frequent promoter hypermethylation of tumor-related genes in head and neck squamous cell carcinoma. Oncol Rep 22: $1519-1526,2009$.

11. Meng W, Huebner A, Shabsigh A, et al: Combined RASSF1A and RASSF $2 A$ promoter methylation analysis as diagnostic biomarker for bladder cancer. Mol Biol Int 2012: 70181, 2012.

12. Foukakis T, Au AY, Wallin G, et al: The Ras effector NOREIA is suppressed in follicular thyroid carcinomas with a PAX8-PPAR $\gamma$ fusion. J Clin Endocrinol Metab 91: 1143-1149, 2006.

13. Nakamura N, Carney JA, Jin L, et al: RASSF1A and NORE1A methylation and $B R A F^{\mathrm{V} 600 \mathrm{E}}$ mutations in thyroid tumors. Lab Invest 85: 1065-1075, 2005.

14. Geli J, Kogner P, Lanner F, et al: Assessment of NORE1A as a putative tumor suppressor in human neuroblastoma. Int J Cancer 123: 389-394, 2008.

15. Hanahan D and Weinberg RA: The hallmarks of cancer: the next generation. Cell 144: 646-674, 2011.

16. Vazquez A, Bond EE, Levine AJ and Bond G: The genetics of the p53 pathway, apoptosis and cancer therapy. Nat Rev Drug Discov 7: 979-987, 2008.

17. Richter AM, Pfeifer GP and Dammann RH: The RASSF proteins in cancer; from epigenetic silencing to functional characterization. Biochim Biophys Acta 1796: 114-128, 2009.

18. Macheiner D, Heller G, Kappel S, et al: NORE1B, a candidate tumor suppressor, is epigenetically silenced in human hepatocellular carcinoma. J Hepatol 45: 81-89, 2006.
19. Calvisi DF, Evert M and Dombrowski F: Pathogenetic and prognostic significance of inactivation of RASSF proteins in human hepatocellular carcinoma. Mol Biol Int 2012: 849874, 2012.

20. Shinmura K, Tao H, Nagura K, et al: Suppression of hydroxyurea-induced centrosome amplification by NORE1A and down-regulation of NORE1A mRNA expression in non-small cell lung carcinoma. Lung Cancer 71: 19-27, 2011.

21. Fernandes MS, Carneiro F, Oliveira C and Seruca R: Colorectal cancer and RASSF family - a special emphasis on RASSF1A. Int J Cancer 132: 251-258, 2013.

22. Macheiner D, Gauglhofer C, Rodgarkia-Dara C, et al: NORE1B is a putative tumor suppressor in hepatocarcinogenesis and may act via RASSF1A. Cancer Res 69: 235-242, 2009.

23. Geli J, Kiss N, Lanner F, et al: The Ras effectors NORE1A and RASSF1A are frequently inactivated in pheochromocytoma and abdominal paraganglioma. Endocr Relat Cancer 14: 125-134, 2007.

24. Lee CK, Lee JH, Lee MG, et al: Epigenetic inactivation of the NORE1 gene correlates with malignant progression of colorectal tumors. BMC Cancer 10: 577, 2010.

25. Guo C, Tommasi S, Liu L, et al: RASSF1A is part of a complex similar to the Drosophila Hippo/Salvador/Lats tumor-suppressor network. Curr Biol 17: 700-705, 2007.

26. Zeng Q and Hong W: The emerging role of the hippo pathway in cell contact inhibition, organ size control, and cancer development in mammals. Cancer Cell 13: 188-192, 2008.

27. Chan SW, Lim CJ, Chen L, et al: The Hippo pathway in biological control and cancer development. J Cell Physiol 226: 928-939, 2011.

28. Avruch J, Praskova M, Ortiz-Vega S, et al: Nore1 and RASSF1 regulation of cell proliferation and of the MST1/2 kinases. Methods Enzymol 407: 290-310, 2006.

29. Ehrkamp A, Herrmann C, Stoll R and Heumann R: Ras and rheb signaling in survival and cell death. Cancers 5: 639-661, 2013.

30. Ng YK, Lau WS, Lui VW, et al: Full-length Mst1 exhibits growth promoting function in human hepatocellular carcinoma cells. FEBS Lett 587: 496-503, 2013.

31. Aoyama Y, Avruch J and Zhang XF: Nore1 inhibits tumor cell growth independent of Ras or the MST1/2 kinases. Oncogene 23: 3426-3433, 2004.

32. Naryzhny SN: Proliferating cell nuclear antigen: a proteomics view. Cell Mol Life Sci 65: 3789-3808, 2008.

33. Brinckerhoff CE, Rutter JL and Benbow U: Interstitial collagenases as markers of tumor progression. Clin Cancer Res 6: 4823-4830, 2000.

34. Trieb K and Kotz R: Proteins expressed in osteosarcoma and serum levels as prognostic factors. Int J Biochem Cell Biol 33: 11-17, 2001

35. Grigorian M and Lukanidin E: Activator of metastasis in cancer cells, Mst1/S100A4 protein binds to tumor suppressor protein p53. Genetika 39: 900-908, 2003 (In Russian). 